\title{
Gewalt gegen Frauen Untersuchungen zum Dekretalenrecht des 12. Jahrhunderts
}

\author{
GiSEla DROSSBACH
}

\section{Einleitung}

Gewalt (vis oder violentia) gegen Frauen gibt es im Mittelalter in vielfältigen Formen, als unmittelbaren Zwang (violentia), wie z. B. bei Sklavenschaft und Vergewaltigung, oder in subtilerer Form als Bedingung für mangelnde Willensfreiheit (vis). Hier geht es vor allem um die letztere Art von Gewalt, um die Missachtung einer Person beim Zustandekommen einer gültigen Ehe bzw. innerhalb einer kirchenrechtlich gültigen Ehe.

Der Kontext, in dem "Gewalt" gegen Frauen thematisiert wird, ist das Kirchenrecht des 12. Jahrhunderts, d. h. die Zeit der Klassischen Kanonistik, eine Zeit großer systematischer Rechtssammlungen, in denen wie in dem um 1140 abgeschlossenen ,Decretum Gratiani ${ }^{\text {" }}$ nach Themen geordnet Fragestellungen (quaestiones) aufgeworfen werden, die mit Hilfe von Referenztexten aus der kirchlichen Tradition (auctoritates) zu einer rechtsverbindlichen Antwort (dictum) geführt werden.' U. a. enthalten sie auch umfangreiche Rubriken zum Eherecht. ${ }^{2}$

Ein neu aufkommender Rechtsbestand sind die unter den juristisch versierten Päpsten Alexander III., Innocenz III. und Gregor IX. ergangenen Dekretalen, Briefe und Urkunden, die päpstliche Entscheidungen in Rechtsfragen darstellen. ${ }^{3}$ Auch sie nehmen im Zusammenhang mit dem kirchlichen Eherecht Bezug auf Gewalt. So finden sich zahlreiche eherechtlich bedeutsame Fälle, in denen auf Gewalt gegen Frauen im Rahmen ehelicher Verhältnisse Bezug genommen wird. Leitendes Interesse bei der Untersuchung soll deshalb im Folgenden sein, ob und inwiefern das kirchliche Recht im 12. Jahrhundert Frauen gegen Gewalt in Schutz nimmt.

Eine solche Fragestellung scheint deshalb berechtigt, da Untersuchungen zum mittelalterlichen kanonischen Recht sich zwar intensiv mit dem Eherecht auseinandersetzen, aber die Gewalt- und Schutzaspekte für Frauen bei dieser Forschung weniger Beachtung finden. ${ }^{4}$

Zum ,Decretum Gratiani“ siehe zuletzt den ausgezeichneten Überblick von Jean Werckmeister, Decretum Gratiani. In: Lexikon für Kirchen- und Staatskirchenrecht. Bd. 1 (2000), S. 375-378.

2 Ein umfassender Traktat über die Ehe findet sich in den Causae 27-36. Hrsg. v. Emil Friedberg, Codex Iuris Canonici. Bd. 1: Decretum Magistri Gratiani. Leipzig 1879, Sp. 1046-1292.

" Vgl. in Auswahl: Peter Landau, Die Entstehung der systematischen Dekretalensammlungen und die europäische Kanonistik des 12. Jahrhunderts. Zeitschrift der Savigny-Stiftung für Rechtsgeschichte kan. Abt. 65 (1979). S. 120-148, wiederabgedruckt in: Peter Landau, Kanones und Dekretalen. Beiträge zur Geschichte der Quellen des kanonischen Rechts (Bibliotheca Eruditorum 2), Goldbach 1997, S. 227*-255*; Gisela Drossbach, Schools and decretals in the $12^{\text {th }}$ century. The Collectio Francofurtana. Bulletin of Medieval Canon Law 24 (2000), S. 69-82; Charles Duggan, Decretals and the creation of new law in the twelfth century. Judges, judgements, equity and law (Collected Studies Series 607). Aldershot u. a. 1998; Charles Duggan, Twelfthcentury decretal collections (University of London Historical Studies 12). London 1963.

4 Der Bereich des Eherechts hat bereits gegen Ende des 19. Jahrhunderts monographische Darstellungen erfahren: Adhémar Esmein, Le mariage en droit canonique. Hrsg. v. Robert Génestal und Jean Dauvillier. 2 Bde. 2. Aufl. Paris 1929-35 (1. Aufl. Paris 1891); Joseph Freisen, Geschichte des kanonischen Eherechts bis zum Verfall der Glossenliteratur. 2. Aufl. Paderbom 1893, Reprint 1963; Jean Dauvillier, Le mariage dans le droit 
In der mediävistischen Sozialgeschichte, insbesondere in der Frauenforschung („Gender Studies"), ist das ein gängigeres Thema, allerdings nicht im Zusammenhang mit der mittelalterlichen Rechtsgeschichte. Das Kirchenrecht als historische Quelle für soziale Belange heranzuziehen, ist im Übrigen nicht nur bei der Erforschung des Mittelalters ungewöhnlich.

Somit geht es im Folgenden nicht um Gewalt gegen Frauen im Allgemeinen und ihre einzelnen Formen, sondern um die Darstellung und Auslegung einiger kirchenrechtlicher Bestimmungen, die sich im 12. Jahrhundert mit Eherechtsfällen beschäftigen und damit auf das Thema "Gewalt" (vis) eingehen.

Es wird zu klären sein, welche Ehekonzepte dabei deutlich werden und wie Frauen Schutz vor Gewalt finden. Dazu möchte ich zunächst kurz auf den Stellenwert des Kirchenrechts im 12. Jahrhundert eingehen.

\section{Das Kirchenrecht im 12. Jahrhundert}

Neben theoretischen Überlegungen sind im 12. Jahrhundert vor allem vier Ebenen der Kirchenrechtsentwicklung zu berücksichtigen:

1. die systematisch rationale Reflexion, wissenschaftliche Distinktion und Erforschung des kirchenrechtlichen Überlieferungsbestandes mit dem Ziel, verbindliche Rechtsbestimmungen zu finden, wie es v. a. im ,Decretum Gratiani“ der Fall ist; ${ }^{5}$

2. das allmähliche machtpolitische Auseinandertreten von sacerdotium und regnum, das mit den Eckpunkten Beilegung des Investiturstreits und Wormser Konkordat (1122) zu kennzeichnen ist ${ }^{6}$ und das sich im Recht als Differenzierung zwischen Kanonistik und Legistik zeigt, wie es z. B. die beiden Fakultäten in Bologna betreiben; ${ }^{7}$

3. die Konzilien, insbesondere das dritte Laterankonzil von 1179, mit seinen auf kirchliche Allgemeinverbindlichkeit abgestellten normativen Festlegungen, wie z. B. durch Papst Alexander III. (1159-1181), der nach dem Frieden von Venedig 1177 die abendländische Kirchenspaltung auf juristischem Weg zu überwinden versucht;

4. die Entwicklung eines neuen Rechts (ius novum), nämlich des Dekretalenrechts in Papstbriefen (epistolae), die allgemein-kirchliche Normativität beanspruchen. ${ }^{8}$ In diesem

classique de l'Église. Depuis le décret de Gratien (1140) jusqu'à la mort de Clément V (1314). Paris 1933. Dann für verschiedene Länder die ehegerichtliche Praxis quellenbedingt v. a. im 14. und 15. Jahrhundert: Rudolf Weigand, Die Rechtsprechung des Regensburger Gerichts in Ehesachen unter besonderer Berücksichtigung der bedingten Eheschließung nach Gerichtsbüchern aus dem Ende des 15. Jahrhunderts. Archiv für katholisches Kirchenrecht 137 (1968), S. 403-463; Richard H. Helmholz. Marriage litigation in medieval England. Cambridge 1974; Christian Schwab, Das Augsburger Offizialatsregister (1348-1352). Ein Dokument geistlicher Diözesangerichtsbarkeit. Edition und Untersuchung (Forschungen zur kirchlichen Rechtsgetschichte und zum Kirchenrecht 25). Köln u. a. 2001; Christiane Deutsch. Ehegerichtsbarkeit im Bistum Regensburg (1480 bis 1538) (Forschungen zur kirchlichen Rechtsgeschichte und zum Kirchenrecht 29). Koln u. a. 2005. Zu prozessualen Fragen des Eherechts nur bei Adhémar Esmein u. Robert Genestal. Le mariage en droit canonique. Bd. 1. 2. Aufl. Paris 1929, S. 449-474; Peter Landau. Papst Innocenz III. und Wilheimines Ehe. Studien zum possessorischen Vertahren im Eherecht. Studia Gratiana 29 (1998). S. 521 - 542.

Vgl. Anm. 1.

6 In Auswahl: Rudolf Schieffer. Investiturstreit. In: Lexikon für Kirchen- und Staatskirchenrecht. Bd. 2 (2002). S. 318; Wemer Goetz, Kirchenreform und Investiturstreit. Stuttgart 2000; Hans Jacobs. Kirchenreform und Hochmittelalter. 3. Aufl. München 1994.

7 Zur „Rechtsschule“ Bologna: Filippo Liotta, Studi di storia del diritto medievale e moderno. Bologna 1999.

8 Siehe Anm. 3. 
Kontext steht auch die kanonistische, auf der Theologie gründende Entwicklung des kirchlichen Eherechts.

\section{Eherechtsfälle}

Für die folgende Untersuchung ist zunächst primär die ,Collectio Francofurtana' heranzuziehen. Sie ist die umfangreichste Dekretalensammlung nach dem ,Decretum Gratiani'. Ihr Autor und ihre Auftraggeber sind bis heute noch nicht bekannt. Sie ist 1181/82 in Troyes in der Champagne oder im nahe gelegenen Sens entstanden und umfasst über 800 Dekretalen und Canones, aufgeteilt in 84 Titel. ${ }^{9}$ Sie entsteht in einer Zeit, in der es noch keine Formvorschriften für die kirchliche Eheschließung gibt und für die man davon ausgehen muss, dass nach kirchlichem Verständnis der Beischlaf (copula carnalis) von Mann und Frau, dem der Ehevertrag nach weltlichen Rechtsgepflogenheiten vorausgeht, die Ehe begründet. ${ }^{10}$

Das Eherecht wird in der ,Collectio Francofurtana' in den ersten neun Titeln abgehandelt. ${ }^{11}$ Es umfasst $u$. a. folgende Bestimmungen: Kinder können bis zum 7. Lebensjahr und Minderjährige bis zum 12. Lebensjahr keine rechtsgültige Ehe schließen, weil ihnen nach allgemeinem Maßstab die körperliche und geistige Reife fehlt. Werden sie dennoch verheiratet, ohne dieses Mindestalter erreicht zu haben, haben sie einen Rechtsanspruch auf Auflösung (dissolutio) bzw. Nichtigerklärung (annulatio) der durch Beischlaf vollzogenen Eheschließung. Darüber hinaus enthält das Eherecht in der ,Collectio Francofurtana weitere Bestimmungen zu sexuellen Vergehen wie Ehebruch, Konkubinat und Vergewaltigung.

\subsection{Erster Fall}

Zunächst wird ein erster Fall nach einer Dekretale Papst Alexanders III. dargestellt, wie er in der ,Collectio Francofurtana' geschildert wird. In der Regel haben alle Texte der ,Collectio Francofurtana ein rubrum, in welcher der Kompilator den rechtlichen Gehalt einer Bestimmung in einem Satz zusammenfasst. Bezüglich dem vorzustellenden Fall lautet das Rubrum: Eine mittels Gewalt (=vis) vertraglich geschlossene Ehe muss aufgelöst werden (dissolvendum est). ${ }^{12}$ Wörtlich heißt es im Text:

Wenn ein Mädchen, das noch nicht 12 Jahre alt ist oder gegen seinen Willen (invita) mit einem Mann zum Beischlaf veranlasst wurde (fuerit copulata) und ihm davon gelaufen ist, sobald es konnte (cum prius potuit), muss dem Mann über eine [Rück-]Fordenung [des Mäd-

Landau [Anm. 3], S. 244*.

10 Eine Überblicksdarstellung des Eherechts vor Einführung des kirchlichen Ehemodells im 12. Jahrhundert bietet Ines Weber, Consensus facit nuptias. Überlegungen zum ehelichen Konsens in normativen Texten des Frühmittelalters. Zeitschrift der Savigny-Stiftung für Rechtsgeschichte kan. Abt. 118 (2001), S. 31-66.

11 Peter Landau u. Gisela Drossbach (Hgg.), Analyse der Collectio Francofurtana. Mit Vorarbeiten von Walther Holtzmann (Monumenta Iuris Canonici). Cittả del Vaticano 2007 (im Druck). Auch der Titel 9 der, Collectio Francofurtana, der sich mit den anschließenden Titeln mit Bestimmungen über Kleriker überschneidet, enthält Eherechtsnormen. Dieser Teil der ,Collectio Francofurtana' stellt gegenüber anderen bekannten Dekretalensammlungen eine beachtliche Zusammenstellung von Eherechtsnormen dar.

12 Frcf. 3.23 [wie Anm. 11]: Matrimonium per vim contractum est dissolvendum. 
chens] Stillschweigen auferlegt werden; und das Mädchen kann dann bei dem Mann bleiben, den es jetzt hat. ${ }^{13}$

Offensichtlich geht es hier um die Ehe zwischen einem Mann und einer Frau, die nach weltlichem Recht durch Vertrag als Muntehe geschlossen wurde. Die so nach weltlichem Recht geschlossene Muntehe bedeutete, dass eine Frau aus der Verfuigungsgewalt ihres Vaters oder ihres Grundherrn in die Verfügungsgewalt des Ehemannes übergeben wurde. ${ }^{14} \mathrm{Bei}$ diesem Rechtsgeschäft galt die Frau als Rechtsgegenstand, nicht als eigenständige Rechtsperson. Die auf diese Weise vertraglich begründete Ehe galt nach kirchlichem Recht als unauflöslich, wenn sie durch Beischlaf vollzogen war.

Wir haben hier den Fall, dass eine junge Frau in einem Verhältnis mit einem Mann steht, in dem sie nicht mehr leben will. Dazu flieht sie aus diesem Verhältnis und lebt mit einem anderen Mann zusammen. Die rechtliche Frage, die sich aus Sicht der Kirche stellt, ist die: Welche von beiden Verbindungen dieser Frau ist eine gültige Ehe? Was wird im Rubrum mit Gewalt (vis) bezeichnet und ist dem Rubrum überhaupt zu glauben, das von einer Auflösung der Ehe (matrimonium est dissolvendum) spricht, zumal doch das katholische Eherecht bis heute keine Scheidung im Sinne einer Wiederheirat bei bestehender kirchenrechtlich gültiger Ehe kennt? ${ }^{15}$

Der Papst entscheidet in der Sache, dass die zweite Beziehung wirksam ist bzw. werden kann. Die im Rubrum genannte vis bezieht sich damit auf die erste Verbindung des Mädchens bzw. der Frau. Denn in dieser ersten Beziehung war die notwendige Willenszustimmung der Frau zur Ehe missachtet worden. Die Frau hatte ihre fehlende Zustimmung kundgetan, indem sie die gleichsam erste Möglichkeit zur Flucht genutzt hatte. Vis ist hier also die Missachtung der notwendigen Willenszustimmung einer Frau zur Ehe.

Im Folgenden soll zur weiteren Klärung des Gesamtzusammenhangs dieser Dekretale ihr Text in einer früheren Sammlung herangezogen werden, nämlich in der ,Collectio Parisien$\operatorname{sis}^{* 16}$

13

Fref. 3.23 [wie Anm. 11]: Si puella nondum XII annum attigit aut invita viro fuerit copulata et cum pritus potuit ab eodem recessit, viro super petitione ipsius post facte silentium imponatis, et illam cum viro quem nunc habet, nisi rationabilis causa obstiterit, faciatis sine contradictione manere.

Zur Dotal- oder Muntehe grundlegend Paul Mikat, Dotierte Ehe - rechte Ehe. Zur Entwicklung des EheschlieBungsrechts in fränkischer Zeit (Rheinisch-westfälische Akademie der Wissenschaften. Geisteswissenschaften G 227). Opladen 1976.

15 Vgl. Robert Battes, Ehescheidung. In: Lexikon für Kirchen- und Staatskirchenrecht. Bd. 1 (2000), S. 539-545.

16 I Par. 176, Die Canonessammlungen zwischen Gratian und Bernhard von Pavia. Hrsg. v. Emil Friedherg. Leipzig 1897 (Neudr. Graz 1956), S. 62: Alex. III. Wigorn. Ep. et abbati de Euesham. Recepta conquestione A. mulieris, quod $W$. filius $R$. causa $H$. I? J filio suo dum ipsam sub custodia sua teneret fecisset impuberem copulari et eam facto illi postmodum remuntiantem cum ad annos discretionis pervenit B. duxisset in uxorem ${ }^{\prime \prime}$ carnaliter cognovisset, cognito etiam quod causa ipsa sub examine fratris vestri Cant. Archiepiscopi discussa fuisset et ad nos appellatione delata d. u. p. s. nostra mandamus ut uraque parte ante te wouts et rer teritute plenius inquisita si taliter inveneritis tum virum quam mulierem ah impetitione memorati ll et filit cus ahwiueritis appellatione cessante et perpetum illis imponentes silentium matrimonium inter $B$.t A. contrachum, nisi causa rationabilis et manifesta impediret, faceretis inviolabiliter observari. Post rectssum autem numit predicatae mulieris nuntius prediciti fili prediciti $W$. ad presentiam nostram accessit asserens. quod predicut. B. uxorem suam ei furatus est causa post appellationem ad nos factam sicut potuit desponsan it. Cumque propter hoc in predicti archiepiscopi presentia utraque pars consisteret testes B. iturati dixerunt predictam mulierem $V$. esse annorum quando filio $W$. fuit desponsata quam citius potuit de custodia illius fugisse et ad etm spontanee accessisse. Testes autem $H$. flii $W$. iurati dixerumt quod XIV. annos haberet quando eam desponsavit et ei post annum et amplius carnaliter sine contradictione adhesit. Allegavit etiam predicus $H$. prefatum 
Eine Frau klagt beim Erzbischof von Canterbury, sie sei als einem Mann zum Schutz Unterstellte mit dessen Sohn als Minderjährige verheiratet worden (fecisset impuberem copulari). Es sei ihr jedoch gelungen zu fliehen. Nachdem sie mündig geworden sei, hätte sie ein anderer Mann zur Frau genommen und sie hätten Beischlaf miteinander gehabt ( $d u$ xisset in uxorem et carnaliter cognovisset). Wann und wie die Frau dem ersten Mann entkommen konnte, wird nicht beschrieben, doch es steht fest, dass sie bei der ersten Eheschließung noch minderjährig war.

Der Erzbischof wendet sich in dieser Angelegenheit an Papst Alexander III. Dieser überträgt dem Bischof von Worcester die Sache zur Entscheidung als delegiertem Richter, gibt aber die Tendenz des Urteils an. Der Papst meint nun in dieser Sache, dass der Bischof von Worcester beide Parteien vorladen solle, Mann und Frau von dem Begehren des Grundherrn und seines Sohnes befreien solle, damit diese ihre Anklage zurückziehen und über die jetzige Verbindung der Frau mit ihrem Mann schweigen. Doch damit war der erste Mann der Frau keinesfalls einverstanden. Er klagte, dass der jetzige Partner der Frau sie ihm geraubt habe (uxorem suam ei furatus est) und dass erst nach der Appellation an den Papst die neue Verbindung entstanden sei.

Infolgedessen werden zwei Zeugenreihen vor dem Bischof vernommen. Die einen sagen, dass die Frau erst fünf Jahre alt gewesen sei, als sie zu der ersten Verbindung gezwungen wurde, und dass sie so schnell wie möglich daraus entflohen (quam citius potuit) und dann freiwillig zum jetzigen Mann gekommen sei. Die anderen Zeugen sagen jedoch aus, die Frau sei bereits vierzehn Jahre alt gewesen, als sie geheiratet habe, und habe ein Ehejahr lang und häufig Beischlaf gehabt, ohne Widerstand zu leisten (ei post annum et amplius carnaliter sine contradictione adhesit). Der verlassene Mann gab zu Protokoll, dass der jetzige Mann die Frau mit Gewalt an sich gebunden und sie sich immer versteckt habe, wenn er selbst sie aufsuchen wollte (sibi predictam mulierem fortiter sublatam iunxisse ipsamque cum requireretur omnino celasse). Da sich dieser Mann im Rechtstreit unterlegen glaubte, appellierte er erneut an Papst Alexander III. Dieser delegierte wiederum den Bischof von Worcester als Richter, schlug aber bereits zwei Lösungswege vor:

Erstens: Wenn die Frau bereits vierzehn oder zwölf Jahre alt war, sie der Verbindung mit dem ersten Mann zugestimmt habe und die copula carnis erfolgt sei, dann ist die erste Ehe wirksam und unauflöslich. Der zweite Lösungsweg ist bereits bekannt. Es ist der oben genannte Satz aus der ,Collectio Francofurtana ${ }^{17}$

Daraus ist zu erkennen, dass drei kumulative Aspekte für eine gültige Eheschließung erforderlich sind:

B. sibi predictam mulierem fortiter sublatam iunxisse ipsamque cum requireretur omnino celasse. Adiecit quoque testes suos testibus $B$. omnimodis prevalere et nullam illis fidem adhiberi debere, quod minus idonei essent utpote furti conscii et omnino incogniti. Cum itaque prefatus $B$. intelligeret sententiam contra se promulgandam ad nostram audientiam appellavit et octabas $S$. Johannis baptistae proximo praeteritas terminum suae appellationi prefixit. Quia igitur rei veritas nequaquam nobis innotuit et cum varia et diversa hinc inde proponuntur nescimus cui partium fides sit potius adhibenda d. u. p. a. s. p. m. quod utraque parte ante vos convocata rei veritatem subtiliter inquiratis et si imveneritis quod predicta mulier XIV. aut XII. esset annorum consensit carnaliterque ei adhesit, predictum B. moneatis et sub anathematis districtione cogatis ut eam ei appellatione cessante restituat et in pace dimittat. Si vero nondum XII. annum attigerat aut invita ei fuerit copulata et quam prius potuit ab eodem recessit illi super repeticione ipsius appellatione cess. silentium imponatis et illam cum eodem B. nisi rationabilis causa obstiterit faciatis sine contradictione manere.

Siehe oben S. $70 \mathrm{f}$. 
1. heiratsfähiges Alter; d. h. Mündigkeit für einen freien Konsensaustausch der Partner;

2. freier Konsens beider Partner;

3. Vollzug des ehelichen Beischlafes (copula carnalis).

Der Bischof hatte nun zunächst das Alter des Mädchens bzw. der Frau zu klären. War das Mädchen zur Zeit der Eheschließung jünger als zwölf Jahre, war die Ehe wegen mangelnder Ehemündigkeit nichtig. War sie jedoch älter als zwölf Jahre und mit der EheschlieBung nicht einverstanden, stellte sich die Frage, ob sie bei erster Gelegenheit weggelaufen war. Wenn sie nicht weggelaufen war, dann bleibt die erste Verbindung bestehen, denn es ist von einem nachträglichen Konsens auszugehen. War die Frau aber bei der ersten Möglichkeit geflohen, so hatte sie faktisch gezeigt, dass sie von Anfang an die Ehe mit diesem Mann nicht wollte, d. h. ein äußerer tatsächlicher Vorgang dient als Indiz für den fehlenden Konsens. In diesem Fall ist die Ehe nicht gültig.

So wird deutlich, dass eine Ehe nach kirchlichem Verständnis nur durch den freien Ehewillen beider Partner zustande kommt, d. h. vor allem auch durch den freien Ehewillen der Frau, die damit kirchlicherseits als Rechtsperson anerkannt wurde. Ist dagegen der freie Wille der Frau zur Ehe und damit zum Beischlaf nicht gegeben, so ist zu folgern, dass die Frau mit Gewalt festgehalten wird. Die kirchlichen Autoritäten treten deshalb für den frei zu leistenden Ehewillen der Frau ein, d. h. sie schützen die Frau vor der Gewalt des Mannes, der sie in einer kirchlich ungültigen Ehe zu halten versucht. Diese Dekretale wurde allerdings nicht in den ,Liber Extra' aufgenommen. Warum das nicht geschah, kann beim momentanen Forschungsstand noch nicht geklärt werden.

\subsection{Zweiter Fall}

Im Folgenden sei eine weitere Dekretale angeführt, die ebenfalls den Zusammenhang von Konsens und Gewalt zum Ausdruck bringt. Der Text stammt wiederum aus der, Collectio Francofurtana'. Er ist in den ,Liber Extra' eingegangen und wird dort mit folgender Rubrik überschrieben: „Nicht jede Gewalt (violentia) behindert eine Ehe“. ${ }^{18}$ Diese Rubrik scheint im Widerspruch zur vorausgegangenen Dekretale zu stehen, wo es heißt: „Ein mit Gewalt (vis) geschlossener Ehevertrag muss aufgelöst werden“". Es wird dann ausgeführt: ${ }^{19}$

Über eine Frau, die gegen ihren Willen mit einem Mann verheiratet wurde und von diesem festgehalten wird, später den Ehekonsens geleistet hat, kann ich nichts Sicheres sagen. da zwischen Gewalt (vis) und Gewalt (vis) ein Unterschied besteht. Sicheres habt Ihr mir darüber später nicht mehr mitgeteilt.

Bei genauerer Betrachtung des Textes steltt sich sein Rechtsgehalt wesentlich weniger eindeutig dar, als das Rubrum vorgibt. Es geht um Gewalt gegen den Willen der Frau, die der Eheschließung vorausgeht. Denn hier handelt es sich wohl um einen Fall, in dem eine Frau gegen ihren Willen an einen Mann übergeben und von diesem gewaltsam festgehalten wird. Was weiter mit diesem Paar geschah, darüber weiß der päpstliche Verfasser der Urkunde wohl nichts Genaues. Offensichtlich ist aber von einem nachträglichen Ehekonsens der Frau die Rede, doch darüber kann der Informant keine sichere Auskunft geben.

18 X 4.1.6 [Anm. 16]: Non omnis violentia impedit contractum matrimonii.

19 Fref. 3.25 [Anm. 11]: De muliere que est imita tradita viro et detenta, quum inter vim et vim sit differentia. et utrum postea consensus intercesserit, certum nobis nihil postea expressisti, nihil certum inde tibi posiumus risspondere. 
Da die ,Collectio Francofurtana' den Text aufnimmt, ist wohl anzunehmen, dass man die Auffassung vertritt, dass sich aus einer unfreiwilligen Ehe durchaus noch eine Konsensehe entwickeln kann. Erst das Rubrum des, Liber Extra' bringt den dahinter stehenden Rechtsgehalt auf den Punkt: Vorausgegangene Gewalt ist kein Hinderungsgrund für eine Konsensehe. Damit wird auch hier wie in der vorangehenden Dekretale deutlich, dass der Konsens der Frau nur auf der Basis ihres freien Willens erfolgen kann. Der freie Wille wird demnach missachtet, wenn die Frau durch einen Mann gewaltsam, d. h. ohne ihre Zustimmung, in eine Ehe gezwungen wird. Zugleich aber wird für möglich gehalten, dass nach einer solchen Gewalttat durchaus noch ein freier Ehekonsens geleistet werden kann.

\section{Burchard von Worms}

Ein weiterer Fall wird dem um das Jahr 1000 entstandenen Dekret des Burchard von Worms entnommen, der in die ,Collectio Francofurtana' Eingang fand. Darin geht es um eine Ehefrau, die außerhalb ihrer Ehe vergewaltigt wurde, um ihren Ehemann, der ihr deswegen nach dem Leben trachtet, und um einen Bischof, der dieser Frau Asyl bietet. ${ }^{20}$

Wenn eine Ehefrau von einem Mann vergewaltigt wurde (uxor consturpata fuerit) und deshalb der Ehemann ihr droht, sie umzubringen, diese aber in Todesangst zum Bischof flüchtet und um Hilfe bittet, muss sich der Bischof mit viel Aufwand darum bemühen, dass sie nicht getötet wird; wenn er das aber nicht kann, darf er sie nicht dem Ehemann übergeben, der sie zurückfordert, denn sie hatte sich ihm [dem Bischof] zum Schutz anvertraut. Vielmehr müsse der Bischof sie dann an einem Ort seiner Wahl überführen, wo sie in Sicherheit leben kann. Wenn aber diese Frau vor Angst zerfressen von dem Ort, den ihr der Bischof zugewiesen hat, flieht, und ihr Mann einmal, zweimal und öfters ihre Herausgabe vom Bischof fordert und verspricht, dass er sie nicht töten wird, hat dies der Bischof zu überprüfen und sie herauszugeben. Wenn der Mann aber nicht verspricht, sie leben zu lassen, dann darf sie der Bischof nicht zurückgeben. Der Mann aber darf dann Zeit seines Lebens keinesfalls eine andere heiraten.

Ein interessanter Fall: Der Bischof gewährt der Vergewaltigten, die bei ihm Schutz sucht, Asyl. Doch die Frau verlässt den sicheren Ort und ist nicht mehr in der Obhut des Bischofs. Für eine solche Situation werden im Text nur zwei von drei Lösungsmöglichkeiten angeführt.

1. Der Mann gibt sein Versprechen, sie nicht zu töten, und sie kehrt zurück.

2. Der Mann verspricht nicht, dass er sie nicht töten würde; sie kehrt nicht zurück, und er darf lebenslang keine neue Ehe schließen.

3. Der Mann könnte argumentieren, sie sei nicht vergewaltigt (uxor consturpata) worden, sondern habe einen Ehebruch (adulterium, fornicatio) begangen und sei verschwunden. Einer Auflösung der Ehe oder einer Wiederverheiratung des Mannes steht das Unauflös-

20 Frcf. 14.6 [Anm. 11]: Si cuius uxor consturpata fuerit, et propterea maritus capitali sententia delere illam machinaverit, ipsa vero, urgente mortis periculo, ad episcopum confugerit, et auxilium quaesierit, operosiori tamen si potest episcopus labore desudet ne occidatur; si vero non potest, nullo modo liceat ei requirenti eam reddere ad occidendum, quae se ei obtulit ad defendendum: sed solerti cura transmittat eam ad locum quem ipse delegerit, ut secura possit vivere. [...] Si autem eadem mulier timore perterrita, a loco ubi eam episcopus destinavit aufugerit, et vir eius ab episcopo semel, aut bis, vel saepius repeterit, si vitam ei obtinere possit, perquirat et reddat: sin cutem, omnino non reddat. Maritus vero, quamdiu ipse vivat, nullo modo alteram chcat. 
lichkeitsgebot entgegen. Solange nicht feststellbar ist, dass die Frau verstorben ist, kann der verlassene Ehemann keine neue gültige Ehe schließen.

Doch die Rubra von drei Handschriften der ,Collectio Francofurtana' lauten: „Wer seine Frau tötet, darf keine andere Frau heiraten. “21 Sie machen deutlich, dass die zweite Lösung im Vordergrund steht, denn für den Mann, der die Ehefrau töten würde, entstünde ein Ehehindernis des Gattenmordes (impedimentum criminis), demzufolge er keine andere Frau heiraten könnte. Hingegen führt das Rubrum von Hs. F lediglich an, dass der Bischof der vergewaltigten und von ihrem Mann tödlich verfolgten Frau Asyl zu gewähren hat. Als weiterer Aspekt von möglicher Gewalt gegen Frauen verhandelt die Rechtssammlung also nicht nur unfreiwilligen Beischlaf, sondern auch die Vergewaltigung (stuprum) außerhalb der Ehe.

\section{Gratian}

\subsection{Vergewaltigung}

In der ,Collectio Francofurtana " wurden bewusst keine Texte aus dem Dekret Gratians aufgenommen, schließlich verstand man die ,Collectio' als ius novum und damit als Fortsetzung des ,alten Rechts“ gegenüber dem Dekret Gratians. ${ }^{22}$ Doch bereits Gratian hat wegweisende Äußerungen zum Thema Gewalt gegen Frauen hervorgebracht. Deshalb sei eines seiner Dicta herangezogen, in dem Gratian nach Abwägungen der Positionen der traditionellen Autoritäten seine eigene Auffassung wiedergibt. In seinem Dictum zur C 32.5.14 wendet er sich gegen eine bestehende Rechtsauffassung, ${ }^{23}$ wonach die Vergewaltigung einer Frau (stuprum) mit dem sexuellen Vergehen des Ehebruchs (adulterium) gleichgesetzt wird: ${ }^{24}$

Wenn also nicht bewiesen werden kann, dass eine Frau, die vergewaltigt wurde, ihre Keusch-
heit verloren hat, dann wird sie keineswegs überführt, dass sie Ehebruch bzw. Unkeuschheit
begangen hätte, wie ja Augustinus in seinem, Liber de bono coniugali“ sagt: "Ehebruch wird
begangen, wenn entweder aus dem Antrieb eigener Begierde oder der Begierde der anderen
Person zustimmend mit der anderen oder dem anderen gegen den Ehevertrag Beischlaf erfolgt,
und auf diese Weise wird die Treue (fides) verletzt, die in den körperlichen und verächtlichen
Sachen das große Gute des Geistes darstellt und der deswegen auch das körperliche Wohl,
aufgrund dessen dieses Leben aufrichtig erhalten wird, zweifelsohne nachgestellt werden
muss.“

Das heißt, dass zum Tatbestand des Ehebruchs die Zustimmung in die Verletzung (violentia) ehelicher Rechte und Pflichten gehört. Demgegenüber ist die Vergewaltigung eine von

21 Frcf. 14.6 [Anm. 11]: Non habeat uxorem, qui suam interficit.

3 Zur zeitgenössischen Anwendung der Begriffe ius vetus und ius novum in der klassischen Kanonistik: Peter Landau, Die Durchsetzung neuen Rechts im Zeitalter des klassischen kanonischen Rechts. In: Gert Melville (Hg.), Institutionen und Geschichte. Theoretische Aspekte und mittelalterliche Befunde (Norm und Struktur 1 ). Köln U. a. 1992, S. $137-155$.

23 Siehe zum Beispiel im fränkischen Recht: Freisen [Anm. 4]. S. 859.

24 Dictum Gratiani ad C 32.5.14 [Anm. 16] Bd. 1, Sp. 1136: Cum ergo hec, que violentium passa est, pudicitium amisisse probari non potest, nullo modo convincitur fornicationis vel adulteril crimen incurrisse. Li enim Augustinus ait in libro de bono coniugali [c. 4]: ,Adulterium conmittitur, cum vel propriae libidinis instinctu. vel alienae consensu cum altera vel altero contra pactum coniugale concumbitur, atque ita frangitur fides, que in rebus corporeis et abiectis magnum animi bonum est, et ideo ei salufem quoque corporalem. qua etiam vita ista continetur, certum est debere postponit. “ 
außen kommende Rechtsverletzung einer Frau, bei der die Keuschheit als innere Einstellung nicht zerstört wird, sondern intakt bleibt. Begeht jedoch ein Ehemann Ehebruch, so ist er unkeusch, denn er handelt aus eigener Begierde bzw. stimmt der Begierde eines anderen zu. Auf diese Weise muss der Mann das Einverständnis der Frau zum außerehelichen Geschlechtsverkehr beweisen. Wenn der Beweis misslingt, wird zugunsten der Frau ihre Keuschheit vermutet. Dass ihre Unschuld vermutet wird, stellt einen Schutz der Frau dar. Denn es ist Aufgabe des Ehemanns zu beweisen, dass sie dem Ehebruch zugestimmt hat.

\subsection{Frauenhaus}

Zum Thema Gewalt gegen Frauen kommt Gratian auch eine Bedeutung auf dem langen Weg zum heutigen „Frauenhaus" zu. Gratian hatte wohl wahrgenommen, dass unliebsame Ehefrauen von ihren Männern zu Hauf einfach verstoßen wurden. Daraus zieht er die Konsequenz, dass eine Verstoßung zunächst vorläufig durch einen kirchlichen Restitutionsakt, d. h. durch Rückversetzung in die eheliche Gemeinschaft, zu beseitigen ist, bevor ein ordnungsgemäßes Verfahren über den Bestand der Ehe durchgeführt werden kann.

Darauf basierend gibt Papst Alexander III. in einer an die Bischöfe von Amiens und Beauvais gerichteten Dekretale die einleuchtende Anweisung, dass man vor der Anordnung der Wiederherstellung der Ehegemeinschaft zunächst Sicherheiten haben müsse, dass der Frau kein Leid von ihrem Mann zugefügt wird. ${ }^{25}$ Wenn der Ehemann aber gegen die Frau einen unbändigen Hass habe, dann solle die Ehegattin der Aufsicht einer bewährten und ehrenhaften Frau an einem Ort anvertraut werden, wo ihr vom Mann und dessen Verwandten keine Gewalt angetan werden kann. Bei dieser Entscheidung zum Schutz der Frau sieht Peter LANDAU bereits im kanonischen Recht des Mittelalters die moderne Institution des Frauenhauses vorweggenommen. ${ }^{26}$

Dies entsprach durchaus sozialer Praxis. Bruce VENARDE hat gerade nachweisen können, ${ }^{27}$ dass vor allem Frauenklöster vergewaltigten Frauen Aufnahme gewähren konnten. Ehemännern, deren verstoßene Frauen nicht zu ihnen zurückkehrten, wurde kirchenrechtlich auferlegt, lebenslang ein enthaltsames Leben zu führen. Diese Dekretale Alexanders III. findet sich nicht in der ,Collectio Francofurtana", wohl aber in einer späteren Sammlung, der ,Compilatio secunda', und hat auf diese Weise Eingang in den ,Liber Extra' gefunden.

\section{Resümee: Kirchliches Eherecht und Schutz der Frau gegen Gewalt}

In den Rechtsquellen des 12. Jahrhunderts wird in vielen Texten und rechtlichen Entscheidungen zunehmend Gewalt (vis) und damit auch sexuelle Gewalt gegen Frauen in differenzierter Weise beschrieben. Ursache hierfür ist, dass in dieser Zeit das Prinzip des Ehekonsens im kirchlichen Recht zur Geltung kommt. Es bewertet den freien Ehewillen sowohl des Mannes wie auch der Frau als ehebegründend. Der Beischlaf ist in diesem Zusammenhang dann nur die Bestätigung für die rechtsgültig eingegangene Ehe aufgrund des freien Ehewillens beider Partner. Insofern wird der im 12. Jahrhundert an den Universitäten in Paris und

JL. $13735=X 2.13 .8$.

Landau. [Anm. 4], S. 529.

Bruce Venarde, Women's monasticism and medieval society. Nunneries in France and England. Ithaca, London 1997, S. $890-1215$. 
Bologna aufgekommene Streit über die Begründung einer rechtsgültigen Ehe entschieden. Durch die kirchliche Ehe erhält die Frau die Möglichkeit, sich aus einer nicht gewollten Ehe zu befreien. Durch ihre Anerkennung als Rechtsperson erhält sie Rechte, die sie auch gegenüber ihrem Mann schützen. Hierin zeigen sich Ansatzpunkte zur Eindämmung der Gewalt in der Ehe.

PD Dr. Gisela Drossbach

Stephan Kuttner Institute of Medieval Canon Law

c'o Leopold-Wenger-Institut fïr Rechtsgeschichte

Luduig-Maximilians-Universität München

Prof. Huber-Platz 2

D-80539 München

Drossbach@jura.uni-muenchen.de 